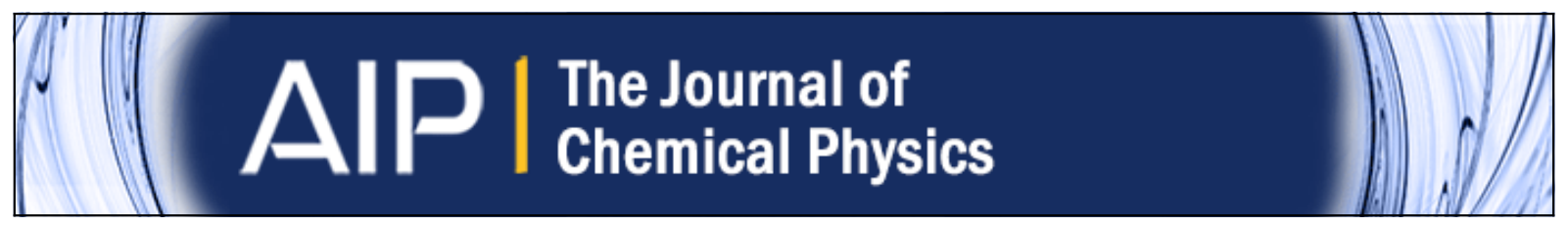

\title{
Structural quantities of quasi-two-dimensional fluids
}

Simon Lang, Thomas Franosch, and Rolf Schilling

Citation: The Journal of Chemical Physics 140, 104506 (2014); doi: 10.1063/1.4867284

View online: http://dx.doi.org/10.1063/1.4867284

View Table of Contents: http://scitation.aip.org/content/aip/journal/jcp/140/10?ver=pdfcov

Published by the AIP Publishing

\section{Articles you may be interested in}

Transient ordering in a quasi-two-dimensional binary liquid near freezing

J. Chem. Phys. 129, 124511 (2008); 10.1063/1.2972982

Structure and thermodynamics of a two-dimensional Coulomb fluid in the strong association regime

J. Chem. Phys. 127, 074501 (2007); 10.1063/1.2764478

Phase separation of a binary two-dimensional core-softened fluid

J. Chem. Phys. 124, 234506 (2006); 10.1063/1.2202852

The structural properties of a two-Yukawa fluid: Simulation and analytical results

J. Chem. Phys. 124, 084501 (2006); 10.1063/1.2166390

Equilibrium Structure of the QuasiTwoDimensional Dipolar Fluid

AIP Conf. Proc. 661, 209 (2003); 10.1063/1.1571314

\section{AlP Re-register for Table of Content Alerts}




\title{
Structural quantities of quasi-two-dimensional fluids
}

\author{
Simon Lang, ${ }^{1,2}$ Thomas Franosch, ${ }^{1,2}$ and Rolf Schilling ${ }^{3}$ \\ ${ }^{1}$ Institut für Theoretische Physik, Leopold-Franzens-Universität Innsbruck, Technikerstraße 25/2, \\ A-6020 Innsbruck, Austria \\ ${ }^{2}$ Institut für Theoretische Physik, Friedrich-Alexander Universität Erlangen-Nürnberg, Staudtstraße 7, \\ 91058 Erlangen, Germany \\ ${ }^{3}$ Institut für Physik, Johannes Gutenberg-Universität Mainz, Staudinger Weg 7, 55099 Mainz, Germany
}

(Received 17 December 2013; accepted 17 February 2014; published online 11 March 2014)

\begin{abstract}
Quasi-two-dimensional fluids can be generated by confining a fluid between two parallel walls with narrow separation. Such fluids exhibit an inhomogeneous structure perpendicular to the walls due to the loss of translational symmetry. Taking the transversal degrees of freedom as a perturbation to an appropriate 2D reference fluid we provide a systematic expansion of the $m$-particle density for arbitrary $m$. To leading order in the slit width this density factorizes into the densities of the transversal and lateral degrees of freedom. Explicit expressions for the next-to-leading order terms are elaborated analytically quantifying the onset of inhomogeneity. The case $m=1$ yields the density profile with a curvature given by an integral over the pair-distribution function of the corresponding $2 \mathrm{D}$ reference fluid, which reduces to its $2 \mathrm{D}$ contact value in the case of pure excluded-volume interactions. Interestingly, we find that the $2 \mathrm{D}$ limit is subtle and requires stringent conditions on the fluid-wall interactions. We quantify the rapidity of convergence for various structural quantities to their $2 \mathrm{D}$ counterparts. (O) 2014 AIP Publishing LLC. [http://dx.doi.org/10.1063/1.4867284]
\end{abstract}

\section{INTRODUCTION}

Confining fluids on length scales comparable to the typical interaction range introduces a competition between local particle near ordering and the layering induced by the particle-wall interaction eventually leading to a dimensional reduction. Of particular interest is the influence of confinement on the structure of fluids, ${ }^{1-9}$ their phase equilibria, equilibrium phase transitions and their critical behavior (see, e.g., Refs. 5, 10-13 and references therein) as well as their dynamic properties. ${ }^{14-21}$ The simplest realization of confinement consists of enclosing the fluid in a slit geometry between two parallel walls. For strong confinement only a few layers of particles fit between the plates. The regime of strong confinement has been investigated by computer simulations (see Refs. 21-27 and references therein) and theoretically by employing suitable closures for the integral equation approaches ${ }^{21,22,24,28-32}$ or within density functional theory. ${ }^{33-36}$

In colloidal suspensions a range of plate separations can be investigated experimentally by imposing a small inclination of one of the plates. ${ }^{16,37-40}$ Alternatively the confinement may be achieved by bringing a large glass sphere in close contact with a planar wall and monitor the colloids confined within the gap. ${ }^{41}$ Structural information on the particle arrangements in confinement has also been collected using small angle $X$-ray scattering, ${ }^{6,42}$ which experimentally allows to probe structural quantities even in the regime of quasi-twodimensional fluids. ${ }^{42,54}$

Recently, it has been shown that in the limit of extreme confinement ${ }^{43}$ a small parameter $n_{0} L^{2}$ emerges, where $n_{0}$ is the number of particles per area and $L$ the separation of the walls. In particular, to leading order the transversal and lat- eral degrees of freedom (dof) decouple, which allows relating equilibrium phase transition lines with respect to the corresponding 2D reference system. ${ }^{43}$ The next-to-leading order of thermodynamic quantities can be elaborated relying on a systematic cluster-expansion. Furthermore, an effective 2D twobody potential is obtained by integrating out the transversal degrees of freedom.

The goal of this work is to elaborate the influence of the transversal dof on structural quantities such as the density profile and the pair-distribution function in the regime of quasi-two-dimensional liquids. Thereby we will elucidate the role of the fluid-fluid and particularly of the fluid-wall interaction. Despite the decoupling property ${ }^{43}$ it will become obvious below that the $2 \mathrm{D}$ limit can be rather subtle, depending on both the physical quantity of interest and the fluid-wall interactions. We will demonstrate that for $L \rightarrow 0$ the coupling between the lateral and transversal dof can be treated as a perturbation which allows expanding the structural entities with respect to their transversal variables. Here, we rely on the recently developed systematic expansion valid for $L \rightarrow 0 .{ }^{43}$ The leading-order term is the corresponding quantity of the homogeneous 2D reference fluid. Particularly, the analytically accessible corrections with respect to the $2 \mathrm{D}$ fluid will be determined. These corrections describe the onset of inhomogeneity for confined fluids emerging from the homogeneous limit of a 2D fluid. The density and the density-density correlation function as probability densities permit to calculate the average of any one-particle and two-particle local observable in the limit of extremely confined liquids.

Our paper is organized as follows. In Sec. II the model is described and various structural quantities are introduced for which the 2D limit will be studied. This limit will be discussed in Sec. III. A summary and conclusions are given in 
Sec. IV. More technical calculations have been transferred to Appendices A-C.

\section{CONFINED FLUIDS: BASIC STRUCTURAL QUANTITIES}

We consider a liquid of $N$ structureless particles between two parallel, planar walls with cross section $A$ and separation $H$. We choose a coordinate system such that the $z$-direction is perpendicular to the walls located at $z= \pm H / 2$. In $x-y$ direction periodic boundary conditions are assumed. Then a point in configurational space is specified by the set of coordinates $\vec{x}$ parallel and perpendicular to the wall $\vec{x}_{i}=\left(\vec{r}_{i}, z_{i}\right)$, $i=1, \ldots N$. The walls are assumed to be impenetrable and the liquid-wall interaction to be additive

$$
U(\{z\} ; L)=\sum_{i=1}^{N} \mathcal{U}\left(z_{i} ; L\right)
$$

with the single-particle-wall interaction

$$
\mathcal{U}(z ; L)= \begin{cases}\infty & \text { for }|z|>L / 2 \\ \mathcal{U}_{-}(L / 2+z)+\mathcal{U}_{+}(L / 2-z) & \text { for }|z| \leq L / 2\end{cases}
$$

Here, we have introduced the effective wall distance $L$ as the transverse length accessible to the particles, and therefore distinguish between point particles and hard spheres

$$
L= \begin{cases}H-\sigma, & \text { hard spheres, } \\ H, & \text { point particles. }\end{cases}
$$

The potential $\mathcal{U}_{+}\left(\mathcal{U}_{-}\right)$is a smooth interaction energy of a fluid particle with the right (left) wall, with possible singularities for vanishing argument. With $\mathcal{U}_{-}(z) \neq \mathcal{U}_{+}(z)$ we further allow for asymmetric wall conditions. The mutual interactions between the fluid particles will be restricted to pair interactions $\mathcal{V}(\vec{x}) \equiv \mathcal{V}(\vec{r}, z)$, only depending on the magnitude $|\vec{x}|$ of their relative distance

$$
V_{0}(\{\vec{x}\})=\sum_{i<j}^{N} \mathcal{V}\left(\vec{x}_{i}-\vec{x}_{j}\right) .
$$

Then the total interaction energy reads

$$
V(\{\vec{x}\} ; L)=V_{0}(\{\vec{x}\})+U(\{z\} ; L) .
$$

In the following we investigate thermal averages using the configurational part of the canonical ensemble $\rho(\{\vec{x}\} ; L)=\exp [-\beta V(\{\vec{x}\} ; L)] / Z$, where $Z(T, A, N, L)$ $=\int\left[\prod_{i=1}^{N} \mathrm{~d}^{2} r_{i} \mathrm{~d} z_{i}\right] \exp [-\beta V(\{\vec{x}\} ; L)]$ denotes the configurational partition function. Integrals over lateral coordinates are performed over the cross section $A$ and the perpendicular coordinates are confined to $-L / 2 \leq z_{i} \leq L / 2, i=1, \ldots, N$. Therefore, the thermodynamic relevant volume is the accessible volume $A L$ and the corresponding number density is given by $n=N / A L$. Canonical averages are indicated by angular brackets $\langle(\cdots)\rangle$. The thermodynamic limit (TD-limit) $N \rightarrow \infty$, $A \rightarrow \infty$ is taken such that the area density $n_{0}=N / A$ remains fixed. Keeping the particle density $n=N / A L$ constant implies that $n_{0}$ converges to zero for $L \rightarrow 0$, i.e., the $2 \mathrm{D}$ system becomes an ideal gas. Therefore, to ensure that correlations between the particles persist in the $2 \mathrm{D}$ limit one has to fix $n_{0}$ instead of $n$. The dependence on the variables $T, A, N$ will mostly be suppressed, while we often highlight the dependence on the effective wall separation $L$.

A basic structural entity characterizing the distribution of particles in a fluid is the $m$-particle density $\rho^{(m)}\left(\vec{r}_{1} z_{1}, \ldots, \vec{r}_{m} z_{m} ; L\right){ }^{44}$ Within the confined geometry as introduced above, the $m$-particle density can be written in the subsequent form

$$
\begin{aligned}
\rho^{(m)} & \left(\vec{r}_{1} z_{1}, \ldots, \vec{r}_{m} z_{m} ; L\right) \\
\quad= & \rho_{\perp}^{(m)}\left(z_{1}, \ldots, z_{m} ; L\right) \tilde{\rho}^{(m)}\left(\vec{r}_{1} z_{1}, \ldots, \vec{r}_{m} z_{m} ; L\right),
\end{aligned}
$$

where we have introduced the $m$-particle density of the transversal degrees of freedom

$$
\begin{aligned}
\rho_{\perp}^{(m)}\left(z_{1}, \ldots, z_{m} ; L\right) & =\prod_{i=1}^{m} \rho_{\perp}^{(1)}\left(z_{i} ; L\right), \\
\rho_{\perp}^{(1)}\left(z_{i} ; L\right) & =\exp \left[-\beta \mathcal{U}\left(z_{i} ; L\right)\right] / z_{\perp}(L), \\
z_{\perp}(L) & =\int \mathrm{d} z \exp [-\beta \mathcal{U}(z ; L)],
\end{aligned}
$$

and a reduced $m$-particle density

$$
\begin{aligned}
& \tilde{\rho}^{(m)}\left(\vec{r}_{1} z_{1}, \ldots, \vec{r}_{m} z_{m} ; L\right)=\mathcal{N}(L) \frac{N !}{(N-m) !} \\
& \quad \times \int\left[\prod_{j=m+1}^{N} d^{2} r_{j} d z_{j} \rho_{\perp}^{(1)}\left(z_{j} ; L\right)\right] \exp \left[-\beta V_{0}(\{\vec{r} z\} ; L)\right] / Z_{\|} .
\end{aligned}
$$

The normalization factor is given by

$$
\mathcal{N}(L)=Z_{\|} Z_{\perp}(L) / Z(L),
$$

with partial partition functions $Z_{\|} \equiv Z_{\|}(T, A, N)$ corresponding to the $2 \mathrm{D}$ reference fluid with pair potential $V_{0}^{\|}\left(\vec{r}_{1}, \ldots, \vec{r}_{m}\right) \equiv V_{0}(\{\vec{r}\}, 0)$ and $Z_{\perp} \equiv Z_{\perp}(T, N, L)=\left(z_{\perp}(L)\right)^{N}$ to the ideal gas of the transversal dof with wall potential $U(\{z\} ; L)$ from Eq. (1). Note that Eq. (6) factorizes the trivial $z_{i}$ - and $L$-dependence of $\rho_{\perp}^{(m)}$ from the nontrivial one of $\tilde{\rho}^{(m)}$. For $m=1$ one obtains the density profile

$$
n(z ; L)=\rho^{(1)}(\vec{r} z ; L),
$$

which is independent of $\vec{r}$ due to translational invariance along the walls. The pair-distribution function $g\left(\vec{r}-\vec{r}^{\prime}, z, z^{\prime} ; L\right)$ is related to $\rho^{(2)}\left(\vec{r} z, \vec{r}^{\prime} z^{\prime} ; L\right)$ by

$$
\begin{gathered}
g\left(\vec{r}-\vec{r}^{\prime}, z, z^{\prime} ; L\right)=\rho^{(2)}\left(\vec{r} z, \vec{r}^{\prime} z^{\prime} ; L\right) / \rho^{(1)}(\vec{r} z ; L) \rho^{(1)}\left(\vec{r}^{\prime} z^{\prime} ; L\right) \\
=\tilde{\rho}^{(2)}\left(\vec{r} z, \vec{r}^{\prime} z^{\prime} ; L\right) / \tilde{\rho}^{(1)}(\vec{r} z ; L) \tilde{\rho}^{(1)}\left(\vec{r}^{\prime} z^{\prime} ; L\right)
\end{gathered}
$$

where have used Eqs. (6) and (7).

A further measure of structural properties is the densitydensity correlation function $G\left(\vec{r}-\vec{r}^{\prime}, z, z^{\prime} ; L\right)$, which can be decomposed into a self part

$$
G^{(s)}\left(\vec{r}, z, z^{\prime} ; L\right)=\frac{1}{n_{0}} \rho^{(1)}(\vec{r} z ; L) \delta(\vec{r}) \delta\left(z-z^{\prime}\right),
$$


and a distinct part

$$
\begin{aligned}
& G^{(d)}\left(\vec{r}, z, z^{\prime} ; L\right) \\
& \quad=\frac{1}{n_{0}}\left[\rho^{(2)}\left(\vec{r} z, \vec{r}^{\prime} z^{\prime} ; L\right)-\rho^{(1)}(\vec{r} z ; L) \rho^{(1)}\left(\vec{r}^{\prime} z^{\prime} ; L\right)\right],
\end{aligned}
$$

i.e.,

$$
\begin{aligned}
G\left(\vec{r}-\vec{r}^{\prime}, z, z^{\prime} ; L\right)= & G^{(s)}\left(\vec{r}-\vec{r}^{\prime}, z, z^{\prime} ; L\right) \\
& +G^{(d)}\left(\vec{r}-\vec{r}^{\prime}, z, z^{\prime} ; L\right) .
\end{aligned}
$$

The total correlation function, $h\left(\vec{r}, z, z^{\prime} ; L\right)=g\left(\vec{r}, z, z^{\prime} ; L\right)$ -1 , and the direct correlation function $c\left(\vec{r}, z, z^{\prime} ; L\right)$ are related by the Ornstein-Zernike equation of inhomogeneous fluids: ${ }^{44,45}$

$$
\begin{aligned}
c\left(\vec{r}, z, z^{\prime}\right)= & h\left(\vec{r}, z, z^{\prime}\right) \\
& -\int \mathrm{d}^{2} r^{\prime \prime} \int \mathrm{d} z^{\prime \prime} c\left(\vec{r}-\vec{r}^{\prime \prime}, z, z^{\prime \prime}\right) n\left(z^{\prime \prime}\right) \\
& \times h\left(\vec{r}^{\prime \prime}-\vec{r}^{\prime}, z^{\prime \prime}, z^{\prime}\right) .
\end{aligned}
$$

It is useful to represent real space functions $f(\vec{r}, z)$ as superpositions of symmetry-adapted Fourier modes ${ }^{46}$

$$
f_{\mu}(\vec{q} ; L)=\int \mathrm{d}^{2} r \mathrm{~d} z f(\vec{r}, z ; L) \exp \left(i Q_{\mu} z\right) \mathrm{e}^{i \vec{q} \cdot \vec{r}},
$$

where the discrete wave numbers $Q_{\mu}=2 \pi \mu / L, \mu \in \mathbb{Z}$ characterize the modulations perpendicular to the walls. Here, $\vec{q}=\left(q_{x}, q_{y}\right)$ are the conventional discrete (for finite $A$ ) wave vectors in the $x-y$-plane. Similarly, for real-space correlation functions $F\left(\vec{r}-\vec{r}^{\prime}, z, z^{\prime} ; L\right)$, we employ the Fourier modes:

$$
\begin{aligned}
F_{\mu \nu}(q ; L)= & \int \mathrm{d}^{2} r \mathrm{~d} z \mathrm{~d} z^{\prime} F\left(\vec{r}, z, z^{\prime} ; L\right) \\
& \times \exp \left(-i Q_{\mu} z\right) \exp \left(i Q_{\nu} z^{\prime}\right) \mathrm{e}^{-i \vec{q} \cdot \vec{r}} .
\end{aligned}
$$

Note, $f_{\mu}(\vec{q} ; L)$ and $F_{\mu \nu}(q ; L)$ depend only on the magnitude $q=|\vec{q}|$ due to the rotational symmetry with respect to the $z-$ axis. The density profile within the slit is decomposed into the discrete wave-numbers:

$$
n_{\mu}(L)=\int \mathrm{d} z \exp \left(i Q_{\mu} z\right) n(z ; L) .
$$

The Fourier transform of the density-density correlation function $G\left(\vec{r}, z, z^{\prime} ; L\right)$ is referred to as generalized structure factor $S_{\mu \nu}(q ; L)$ with corresponding decomposition into self and distinct part $S_{\mu \nu}(q ; L)=S_{\mu \nu}^{(s)}(q ; L)+S_{\mu \nu}^{(d)}(q ; L)$. Explicitly one infers

$$
\begin{gathered}
S_{\mu \nu}^{(s)}(q ; L)=n_{\nu-\mu}(L) / n_{0}, \\
S_{\mu \nu}^{(d)}(q ; L)=\int \mathrm{d}^{2} r \mathrm{~d} z \mathrm{~d} z^{\prime} G^{(d)}\left(\vec{r}, z, z^{\prime} ; L\right) \\
\\
\times \exp \left(-i Q_{\mu} z\right) \exp \left(i Q_{\nu} z^{\prime}\right) \mathrm{e}^{-i \vec{q} \cdot \vec{r}} .
\end{gathered}
$$

The transform $c_{\mu \nu}(q ; L)$ of the direct correlation functions is related to $S_{\mu \nu}(q ; L)$ via the inhomogeneous OrnsteinZernike equation, ${ }^{45}$ which we reformulate in terms of the symmetry-adapted modes ${ }^{46}$

$$
\mathbf{S}^{-1}(q ; L)=\frac{n_{0}}{L^{2}}[\mathbf{v}(L)-\mathbf{c}(q ; L)]
$$

with the following matrix notation $[\mathbf{c}(q ; L)]_{\mu \nu}=c_{\mu \nu}(q ; L)$, etc. The Fourier modes $v_{\mu \nu}(L) \equiv v_{\nu-\mu}(L)$ of the local volume $v(z)=1 / n(z)$ are related to $n_{\mu \nu}(L) \equiv n_{v-\mu}(L)$ by

$$
\mathbf{v}(L) \mathbf{n}(L)=L^{2} \mathbf{1}
$$

see Refs. 17 and 46.

\section{TWO-DIMENSIONAL LIMIT}

In Sec. III A we discuss conditions on the interactions such that the $m$-particle density and related correlation functions converge for $L \rightarrow 0$ properly to their corresponding 2D counterparts. Then, we elaborate a systematic expansion of the structural quantities as introduced in Sec. II. To avoid cumbersome notation, quantities depending only on the 2D vectors $\vec{r}$ or $\vec{q}$ will be considered as obtained from a canonical average with total interaction potential $V_{0}(\{\vec{r}\}) \equiv V_{0}(\{\vec{r}\},\{0\})$. For instance, $\rho^{(m)}\left(\vec{r}_{1}, \ldots, \vec{r}_{m}\right)$ denotes the 2D $m$-particle density and

$$
\begin{gathered}
G(\vec{r})=G^{(s)}(\vec{r})+G^{(d)}(\vec{r}), \\
G^{(s)}(\vec{r})=\frac{1}{n_{0}} \rho^{(1)}(\vec{r}) \delta(\vec{r}) \equiv \delta(\vec{r}), \\
G^{(d)}\left(\vec{r}-\vec{r}^{\prime}\right)=\frac{1}{n_{0}}\left[\rho^{(2)}\left(\vec{r}, \vec{r}^{\prime}\right)-\rho^{(1)}(\vec{r}) \rho^{(1)}\left(\vec{r}^{\prime}\right)\right],
\end{gathered}
$$

refers to the density-density correlation function of the corresponding 2D fluid decomposed into the self and distinct part. Of course, $G(\vec{r}), G^{(s)}(\vec{r})$, and $G^{(d)}(\vec{r})$ depend on $|\vec{r}|=r$, only. The planar total correlation function $h(r)$ is connected to the distinct part of the density-density correlation function by $G^{(d)}(r)=n_{0} h(r)$ and the 2D pair-distribution function follows by the relation $g(r)=1+h(r)$.

\section{A. Existence of the two-dimensional limit}

The static structure of the confined fluid converges properly to the 2D limit if the fluid becomes structureless in the transverse direction. As will be demonstrated below this requires certain conditions on the microscopic interactions of the particles with the walls, i.e., the approach to the planar limit depends on qualitative features of $\mathcal{U}_{ \pm}(z)$ which determine the wall potential $\mathcal{U}(z ; L)$ (cf. Eq. (2)). To discuss convergence with respect to different wall separations of functions $f\left(\vec{r}_{1} z_{1}, \vec{r}_{2} z_{2}, \ldots ; L\right)$ defined on the slit, it will be convenient to establish the dimensionless transversal position $\tilde{z}=z / L$, with the fixed domain $\tilde{z} \in[-1 / 2,1 / 2]$ as fundamental variable. Then, this function has a proper 2D limit if $f\left(\vec{r}_{1} \tilde{z}_{1} L, \vec{r}_{2} \tilde{z}_{2} L, \ldots ; L\right)$ becomes independent of $\tilde{z}_{i}$ for $L \rightarrow 0$. As an example we consider the density profile. From Eq. (19), we obtain

$$
n_{\mu}(L)=L \int_{-1 / 2}^{1 / 2} \mathrm{~d} \tilde{z} n(\tilde{z} L ; L) \exp (2 \pi i \mu \tilde{z}) .
$$

The proper $2 \mathrm{D}$ limit requires that all non-trivial modes vanish, $n_{\mu}(L) \rightarrow n_{0} \delta_{\mu 0}$ for $L \rightarrow 0$ which is satisfied if $n(\tilde{z} L ; L)$ $\rightarrow n_{0} / L$, i.e., the density profile for $L \rightarrow 0$ becomes independent of the scaled transversal coordinate $\tilde{z}$. For a hard-sphere 
liquid with neutral walls this is demonstrated in Fig. 1 of Ref. 21. This convergence describes a fluid for $L \rightarrow 0$, which is determined by the lateral interaction potential $V_{0}(\{\vec{r}\})$ only.

Since the $m$-particle density $\rho^{(m)}\left(\vec{r}_{1} z_{1}, \ldots, \vec{r}_{m} z_{m} ; L\right)$ is entirely determined by the densities $\rho_{\perp}^{(1)}(z ; L)$ and $\tilde{\rho}^{(m)}\left(\vec{r}_{1} z_{1}, \ldots, \vec{r}_{m} z_{m} ; L\right)$ (cf. Eq. (6)), the convergence to the $2 \mathrm{D}$ limit requires both densities to become independent of $\tilde{z}$ and $\tilde{z}_{1}, \ldots, \tilde{z}_{m}$, respectively. As will be demonstrated in Subsection III B, the reduced $m$-particle density $\tilde{\rho}^{(m)}\left(\vec{r}_{1} \tilde{z}_{1} L, \ldots, \vec{r}_{m} \tilde{z}_{m} L ; L\right)$ converges properly for $L \rightarrow 0$, which is not necessarily the case for $\rho_{\perp}^{(1)}(\tilde{z} L ; L)$. Since $\rho_{\perp}^{(1)}(\tilde{z} L ; L)$ depends only on $\mathcal{U}(\tilde{z} L ; L)$, its convergence is solely controlled by the particle-wall interaction.

Assume that the wall potential for fixed $\tilde{z}$ fulfills the smoothness criterion

$$
\mathcal{U}(z=\tilde{z} L ; L)-\mathcal{U}(0 ; L)=\mathcal{O}(L)
$$

which is valid for potentials that are analytic in $z$. Then the "bare" density profile $\rho_{\perp}^{(1)}(z ; L)$ becomes flat even on the scale of the plate distance:

$$
\rho_{\perp}^{(1)}(\tilde{z} L ; L)=\frac{1}{L}[1+\mathcal{O}(L)]
$$

This in turn implies convergence of the Fourier modes of the density profile:

$$
n_{\mu}(L)=n_{0}\left[\delta_{\mu 0}+\left(1-\delta_{\mu 0}\right) \mathcal{O}(L)\right]
$$

Note, that the normalization of $n(z ; L)$ implies $n_{\mu=0}(L)=N / A$ $=n_{0}$ for all $L$. Thus, the property that only the zero Fourier mode of the density profile (1-particle density) survives in the limit of $L \rightarrow 0$ serves as a definition of proper convergence to a two-dimensional fluid.

It is instructive to give a counterexample for a wall potential such that the smoothness criterion is violated and $n_{\mu}(L)$ does not converge to $n_{0} \delta_{\mu 0}$. For instance for symmetric and repulsive walls with $\mathcal{U}_{ \pm}(z) \equiv \mathcal{U}_{\mathrm{w}}(z)=a z^{-\alpha}, a>0, \alpha>0$, the Boltzmann factor becomes

$$
\begin{aligned}
& \mathrm{e}^{-\beta[\mathcal{U}(\tilde{z} L ; L)-\mathcal{U}(0 ; L)]} \\
& \quad=\exp \left\{-\beta a(L / 2)^{-\alpha}\left[(1-2 \tilde{z})^{-\alpha}+(1+2 \tilde{z})^{-\alpha}-2\right]\right\} \\
& \quad \rightarrow \begin{cases}1 & \text { for } \tilde{z}=0 \\
0 & \text { else }\end{cases}
\end{aligned}
$$

as $L \rightarrow 0$. Hence, the density profile $n(z=\tilde{z} L ; L) \rightarrow 0$ for $\tilde{z} \neq 0$, yet by normalization $\int n(z ; L) \mathrm{d} z=n_{0}$. Thus, we have demonstrated that in this case the density profile becomes singular,

$$
n(z=\tilde{z} L ; L) \rightarrow\left(n_{0} / L\right) \delta(\tilde{z})
$$

in strong contrast to Eq. (29). Equivalently, the Fourier modes of the density profile converge as $n_{\mu}(L) \rightarrow n_{0}$ for all $\mu$. Therefore, the density profile and consequently as well the $m$ particle density do not have a proper 2D limit for this kind of wall potentials. Note that, e.g., Lennard-Jones and Coulomb potentials belong to the class of wall potentials, for which no proper convergence can be achieved.

\section{B. The $m$-particle density and the density profile}

In this subsection we elaborate an expansion of the $m$ particle density with respect to the wall separation $L$. First, we derive asymptotically exact results for smooth pair potentials, followed by an outline of the corresponding result in the case of hard-core interactions. The 1-particle density, i.e., the density profile, the most basic quantity characterizing inhomogeneous fluids, will be discussed in more detail.

In Ref. 43 it has been demonstrated that the thermodynamic behavior of quasi-two-dimensional fluids can be obtained from a systematic expansion around a 2D reference fluid. This is based on the fact that the transverse coordinates $z_{i}$ are of order $L$. For smooth pair potentials we use the expansion

$$
\left|\vec{x}_{i}-\vec{x}_{j}\right|=r_{i j}+z_{i j}^{2} / 2 r_{i j}+\mathcal{O}\left(z_{i j}\right)^{4},
$$

with $r_{i j}=\left|\vec{r}_{i}-\vec{r}_{j}\right|$ and $z_{i j}=\left(z_{i}-z_{j}\right)$. Then we obtain from Eq. (4),

$$
\begin{aligned}
\exp \left[-\beta V_{0}(\{\vec{x}\})\right]= & \exp \left[-\beta V_{0}(\{\vec{r}\})\right] \\
& \times\left[1+\sum_{l=1}^{\infty} \sum_{i<j} v_{l}\left(\vec{r}_{i} z_{i}, \vec{r}_{j} z_{j}\right)\right],
\end{aligned}
$$

where

$$
v_{l}\left(\vec{r}_{i} z_{i}, \vec{r}_{j} z_{j}\right)= \begin{cases}-\beta \mathcal{V}^{\prime}\left(r_{i j}\right) z_{i j}^{2} / 2 r_{i j}=\mathcal{O}\left(L^{2}\right) & \text { if } l=1 \\ \mathcal{O}\left(L^{2 l}\right) & \text { if } l>1\end{cases}
$$

i.e., $v_{l}=\mathcal{O}\left(L^{2 l}\right)$ for $l \geq 1$. Note, that the explicit expressions for $v_{l}\left(\vec{r}_{i} z_{i}, \vec{r}_{j} z_{j}\right)$ become more and more involved with increasing $l$, since Eq. (33) has to be extended up to order $\left(z_{i j}\right)^{2 l}$.

Here, a comment is in order. If the particles are charged such that they are interacting via Coulomb forces one might be tempted to use for the corresponding interaction potential of the 2D reference fluid the 2D Coulomb potential: $\sim \ln r_{i j}$. This does not apply here, since the confined fluid even in the 2D limit is embedded in 3D space. Accordingly, the corresponding 2D potential of the reference fluid in this case is the conventional Coulomb potential proportional to $1 / r_{i j}$.

In the following we restrict the expansion of the $m$ particle density to first order in $L^{2}$. In this case the calculations simplify due to the factorization of $v_{1}\left(\vec{r}_{i} z_{i}, \vec{r}_{j} z_{j}\right)$ :

$$
\begin{aligned}
v_{1}\left(\vec{r}_{i} z_{i}, \vec{r}_{j} z_{j}\right) & =v_{1}^{\|}\left(\vec{r}_{i}, \vec{r}_{j}\right) v_{1}^{\perp}\left(z_{i}, z_{j}\right), \\
v_{1}^{\|}\left(\vec{r}_{i}, \vec{r}_{j}\right) & =-\beta \mathcal{V}^{\prime}\left(r_{i j}\right) / 2 r_{i j}, \\
v_{1}^{\perp}\left(z_{i}, z_{j}\right) & =\left(z_{i j}\right)^{2} .
\end{aligned}
$$

The calculation of higher-order terms is straightforward, but cumbersome. Since $\rho_{\perp}^{(m)}\left(z_{1}, \ldots, z_{m} ; L\right)$ is explicitly known a priori for a given particle-wall interaction $\mathcal{U}(z ; L)$, the expansion has to be carried out for the reduced $m$-particle density $\tilde{\rho}^{(m)}\left(\vec{r}_{1}, z_{1}, \ldots, \vec{r}_{m} z_{m} ; L\right)$ only. Equations (8) and (34) lead to

$$
\tilde{\rho}^{(m)}\left(\vec{r}_{1} z_{1}, \ldots, \vec{r}_{m} z_{m} ; L\right)=\sum_{l=0}^{\infty} \tilde{\rho}_{l}^{(m)}\left(\vec{r}_{1} z_{1}, \ldots, \vec{r}_{m} z_{m} ; L\right),
$$


where $\tilde{\rho}_{l}^{(m)}=\mathcal{O}\left(L^{2 l}\right)$. The explicit evaluation of the leading order and its first correction has been transferred to Appendix A. Employing Eq. (36) and defining averages with respect to the perpendicular ensemble $\left\langle f\left(z_{1}, \ldots, z_{k}\right)\right\rangle_{\perp}$ $:=\int d z_{1} \ldots \int d z_{k} f\left(z_{1}, \ldots, z_{k}\right) \rho_{\perp}^{(1)}\left(z_{1}\right) \ldots \rho_{\perp}^{(1)}\left(z_{k}\right)$, we obtain from Eq. (A13) to leading order the 2D $m$-particle density

$$
\tilde{\rho}_{0}^{(m)}\left(\vec{r}_{1} z_{1}, \ldots, \vec{r}_{m} z_{m} ; L\right)=\rho_{\|}^{(m)}\left(\vec{r}_{1}, \ldots, \vec{r}_{m}\right),
$$

and for the leading correction

$$
\begin{aligned}
\tilde{\rho}_{1}^{(m)}\left(\vec{r}_{1} z_{1}, \ldots, \vec{r}_{m} z_{m} ; L\right) & =\sum_{1 \leq i<j \leq m} v_{1}^{\perp}\left(z_{i}, z_{j}\right) v_{1}^{\|}\left(\vec{r}_{i}, \vec{r}_{j}\right) \rho_{\|}^{(m)}\left(\vec{r}_{1}, \ldots, \vec{r}_{m}\right) \\
& +\sum_{i=1}^{m}\left\langle v_{1}^{\perp}\left(z_{i}, z_{m+1}\right)\right\rangle_{\perp} \int d^{2} r_{m+1} v_{1}^{\|}\left(\vec{r}_{i}, \vec{r}_{m+1}\right) \rho_{\|}^{(m+1)}\left(\vec{r}_{1}, \ldots, \vec{r}_{m+1}\right) \\
& +\frac{1}{2}\left\langle v_{1}^{\perp}\left(z_{m+1}, z_{m+2}\right)\right\rangle_{\perp} \int d^{2} r_{m+1} \int d^{2} r_{m+2} v_{1}^{\|}\left(\vec{r}_{m+1}, \vec{r}_{m+2}\right) \\
& \times\left[\rho_{\|}^{(m+2)}\left(\vec{r}_{1}, \ldots, \vec{r}_{m+2}\right)-\rho_{\|}^{(m)}\left(\vec{r}_{1}, \ldots, \vec{r}_{m}\right) \rho_{\|}^{(2)}\left(\vec{r}_{m+1}, \vec{r}_{m+2}\right)\right] \\
& -\left\langle v_{1}^{\perp}\left(z_{m+1}, z_{m+2}\right)\right\rangle_{\perp}\left(\frac{n_{0}^{2} \kappa_{T}}{2 \beta}\right)\left(\left.\frac{\partial}{\partial n_{0}}\right|_{T} \rho_{\|}^{(m)}\left(\vec{r}_{1}, \ldots, \vec{r}_{m}\right)\right) \\
& \times\left.\int d^{2} r_{m+2} v_{1}^{\|}\left(\vec{r}_{m+1}, \vec{r}_{m+2}\right) \frac{\partial}{\partial n_{0}}\right|_{T} \rho_{\|}^{(2)}\left(\vec{r}_{m+1}, \vec{r}_{m+2}\right) .
\end{aligned}
$$

The last term in Eq. (39) results from the thermodynamic limit (cf. Ref. 47). Its integral term does not depend on $\vec{r}_{m+1}$ due to lateral translational invariance. Here, $\kappa_{T}=\left[n_{0} \partial \Sigma /\left.\partial n_{0}\right|_{T}\right]^{-1}$ refers to the $2 \mathrm{D}$ isothermal compressibility of the fluid with surface tension given by $\Sigma=n_{0} k_{B} T+k_{B} T \partial \ln \left(Z_{\|}\right) /\left.\partial A\right|_{T, N}$. The density derivatives of $\rho_{\|}^{(2)}$ and $\rho_{\|}^{(m)}$ can be expressed by $\rho_{\|}^{(k)} \cdot 48$

$$
\begin{aligned}
\left.\frac{\partial}{\partial n_{0}}\right|_{T} \rho_{\|}^{(k)}\left(\vec{r}_{1}, \ldots, \vec{r}_{k}\right) \\
=\left\{k \rho_{\|}^{(k)}\left(\vec{r}_{1}, \ldots, \vec{r}_{k}\right)+\int d^{2} r_{k+1}\left[\rho_{\|}^{(k+1)}\left(\vec{r}_{1}, \ldots, \vec{r}_{k+1}\right)\right.\right. \\
\left.\left.\quad-\rho_{\|}^{(k)}\left(\vec{r}_{1}, \ldots, \vec{r}_{k}\right) \rho_{\|}^{(1)}\left(\vec{r}_{k+1}\right)\right]\right\} /\left(n_{0}^{2} \beta^{-1} \kappa_{T}\right)
\end{aligned}
$$

Additionally, one can prove

$$
\begin{aligned}
& \tilde{\rho}_{1}^{(m)}\left(\vec{r}_{1} z_{1}, \ldots, \vec{r}_{m} z_{m} ; L\right) \\
& \quad \rightarrow \tilde{\rho}_{0}^{(k)}\left(\vec{r}_{1} z_{1}, \ldots, \vec{r}_{k} z_{k} ; L\right) \tilde{\rho}_{1}^{(m-k)}\left(\vec{r}_{k+1} z_{k+1}, \ldots, \vec{r}_{m} z_{m} ; L\right) \\
& \quad+\tilde{\rho}_{1}^{(k)}\left(\vec{r}_{1} z_{1}, \ldots, \vec{r}_{k} z_{k} ; L\right) \tilde{\rho}_{0}^{(m-k)}\left(\vec{r}_{k+1} z_{k+1}, \ldots, \vec{r}_{m} z_{m} ; L\right),
\end{aligned}
$$

for $\vec{r}_{i}, i=1, \ldots, k$ fixed and $\left|\vec{r}_{j}\right| \rightarrow \infty, j=k+1, \ldots, m$. This property preserves the factorization of the $m$-particle density $^{49}$ to first order if a subset of particles $(1, \ldots, k)$ is fixed and the remaining subset $(k+1, \ldots, m)$ is moved to infinity. Further, the result of Eq. (39) formally has the same structure as the first-order correction of the pair-distribution function of a bulk fluid perturbed by an additional pair potential. ${ }^{47} \mathrm{How}-$ ever, Eq. (39) is valid for a 2D fluid perturbed by the translational dof and holds for all $m$.

In the case of hard-sphere interactions a corresponding expansion can be performed by utilizing the cluster expansion derived in Ref. 43. There, a 2D liquid of hard disks of reduced diameter $\sigma_{L}=\left(\sigma^{2}-L^{2}\right)^{1 / 2}$ has been chosen as reference fluid and the cluster function,

$$
\begin{aligned}
f_{i j} & \equiv f\left(r_{i j}, z_{i}, z_{j}\right) \\
& =\Theta\left[r_{i j}^{2}+\left(z_{i}-z_{j}\right)^{2}-\sigma^{2}\right]-\Theta\left(r_{i j}^{2}-\sigma_{L}^{2}\right),
\end{aligned}
$$

has been introduced, where $\Theta(x)$ refers to the Heaviside function. The cluster function satisfies the identity ${ }^{43}$

$$
\begin{aligned}
\exp \left[-\beta V_{0}(\{\vec{x}\})\right] & =\exp \left[-\beta W_{0}(\{\vec{r}\})\right] \prod_{i<j}\left(1+f_{i j}\right) \\
& =\exp \left[-\beta W_{0}(\{\vec{r}\})\right]\left[1+\sum_{i<j} f_{i j}+\ldots\right],
\end{aligned}
$$

where $\exp \left[-\beta W_{0}(\{\vec{r}\})\right]=\prod_{i<j} \Theta\left(r_{i j}^{2}-\sigma_{L}^{2}\right)$ is the Boltzmann factor of the hard disk fluid. Since the first-order term $\sum_{i<j} f_{i j}$ corresponds to $\sum_{i<j} v_{1}\left(\vec{r}_{i} z_{i}, \vec{r}_{j} z_{j}\right)$ in Eq. (34), one obtains $\tilde{\rho}^{(m)}$ for hard-core interactions from Eq. (A8) by the replacement $v_{1} \rightarrow f$. The integrations over $z_{i}, z_{j}$ and $\vec{r}_{i}, \vec{r}_{j}$ can still be disentangled, see Appendix $\mathrm{C}$ for an example. As a result the $m$-particle density $\tilde{\rho}^{(m)}$ for $m=0,1$ is expressed by $\rho_{\|}^{(m)}$, the $m$-particle density of hard disks with reduced diameter $\sigma_{L}$. To eliminate the dependence of $L$ of the reference fluid, a further expansion with respect to $L$ is required in order to obtain the corrections with respect to a 2D-reference fluid with diameter $\sigma$. Beyond the first order one cannot follow the expansion, Eq. (34), by a simple replacement, but it becomes necessary to perform a cluster expansion as elaborated in Ref. 43. Restricting the expansion to zero and first-order corrections allows to determine $\tilde{\rho}^{(m)}, m=0,1$ directly from Eq. (39) by choosing for $\mathcal{V}\left(r_{i j}\right)$ the hard disk potential with a hard-core diameter $\sigma$, replacing $-\left[\beta \mathcal{V}^{\prime}\left(r_{i j}\right) / 2 r_{i j}\right] \rho_{\|}^{(m)}\left(\ldots, \vec{r}_{i}, \ldots, \vec{r}_{j}, \ldots\right)$ by $\left(\exp \left[\beta \mathcal{V}\left(r_{i j}\right)\right] / 2 r_{i j}\right) \rho_{\|}^{(m)}\left(\ldots, \vec{r}_{i}, \ldots, \vec{r}_{j}, \ldots\right) \mathrm{d}\left(\exp \left[-\beta \mathcal{V}\left(r_{i j}\right)\right]\right)$ $/ \mathrm{d} r_{i j}$ and subsequently taking the limit $\lim _{r_{i j} \rightarrow \sigma^{+}} \mathrm{d} \exp \left[-\beta \mathcal{V}\left(r_{i j}\right)\right] / \mathrm{d} r_{i j}=\delta\left(r_{i j}-\sigma^{+}\right)$. This strategy is demonstrated below for the density profile. The firstorder correction, Eq. (39), strongly simplifies for $m=1$ (see Appendix B). One obtains with $\rho_{\|}^{(2)}(r)=n_{0}^{2} g(r)$ from Eq. (B5),

$$
\begin{aligned}
n(z ; L) \equiv & \rho^{(1)}(\vec{r} z ; L) \\
= & n_{0} \rho_{\perp}^{(1)}(z ; L)\left\{1+\left[\left\langle v_{1}^{\perp}\left(z, z_{2}\right)\right\rangle_{\perp}-\left\langle v_{1}^{\perp}\left(z_{2}, z_{3}\right)\right\rangle_{\perp}\right]\right. \\
& \left.\times n_{0} \int d^{2} r^{\prime} v_{1}^{\|}\left(0, \vec{r}^{\prime}\right) g\left(r^{\prime}\right)+\mathcal{O}\left(L^{4}\right)\right\}
\end{aligned}
$$

with $g(r)$ the pair-distribution function of the 2D reference fluid. Note that the average $\left\langle v_{1}^{\perp}\left(z, z_{2}\right)\right\rangle_{\perp}$ is taken with respect to $z_{2}$ only. Since $\int d z \rho_{\perp}^{(1)}(z ; L)\left[\left\langle v_{1}^{\perp}\left(z, z_{2}\right)\right\rangle_{\perp}\right.$ $\left.-\left\langle v_{1}^{\perp}\left(z_{2}, z_{3}\right)\right\rangle\right]=0$ the first-order correction does not contribute to the normalization $\int \mathrm{d} z n(z ; L)=n_{0}$. Substituting $v_{1}^{\perp}$ and $v_{1}^{\|}$from Eq. (36) into Eq. (44), one obtains the explicit 
$z$-dependence

$$
\begin{aligned}
n(z ; L)= & n_{0} \rho_{\perp}^{(1)}(z ; L)\left[1+\pi n_{0} C\left\{z^{2}-2 z\left\langle z_{1}\right\rangle_{\perp}+\left\langle z_{1}^{2}\right\rangle_{\perp}\right.\right. \\
& \left.\left.-\left\langle\left(z_{1}-z_{2}\right)^{2}\right\rangle_{\perp}\right\}+\mathcal{O}\left(L^{4}\right)\right]
\end{aligned}
$$

where the prefactor

$$
C=-\beta \int_{0}^{\infty} \mathrm{d} r \mathcal{V}^{\prime}(r) g(r)
$$

characterizes the first-order corrections. Since the curly bracket in Eq. (45) is of order $\mathcal{O}\left(L^{2}\right)$ the correction is proportional to $n_{0} L^{2}$, the smallness parameter identified in Ref. 43.

Following the procedure as explained above the prefactor $C$ becomes in the case of pure hard-core interactions:

$$
C=g\left(\sigma^{+}\right) \text {. }
$$

If the potentials $\mathcal{U}_{ \pm}(z)$ are analytic at $z=0$, we obtain from Eq. (7):

$$
\rho_{\perp}^{(1)}(z ; L)=\frac{1}{L}\left\{1-\beta\left(\mathcal{U}_{-}^{\prime}(0)-\mathcal{U}_{+}^{\prime}(0)\right) z+\mathcal{O}(L z)+\mathcal{O}\left(z^{2}\right)\right\} .
$$

In the case of symmetric walls, i.e., $\mathcal{U}_{+}(z) \equiv \mathcal{U}_{-}(z)$, it is $\left\langle z_{1}\right\rangle_{\perp}$ $=0$ and it follows from Eqs. (45) and (48) that the profile is parabolic. Consequently, the first-order correction of the profile is of order $\mathcal{O}\left(L^{2}\right)$. For asymmetric walls and $L \rightarrow 0$ the density $n(z ; L)$ is dominated by the bare profile $\rho_{\perp}^{(1)}(z ; L)$, which is linear, i.e., the leading order correction is of order $\mathcal{O}(L)$. Hence, the first-order correction depends qualitatively on the symmetry of the wall-potential. This is as well reflected in the Fourier modes of the density profile

$$
n_{\mu}(L)= \begin{cases}n_{0}=N / A & \text { for } \mu=0, \\ \mathcal{O}\left(L^{\gamma}\right) & \text { else },\end{cases}
$$

and of the local volume

$$
v_{\mu}(L)=\frac{L^{2}}{n_{0}} \begin{cases}{\left[1+\mathcal{O}\left(L^{2 \gamma}\right)\right]} & \text { for } \mu=0, \\ \mathcal{O}\left(L^{\gamma}\right) & \text { else, }\end{cases}
$$

where $\gamma=1$ for asymmetric and $\gamma=2$ for symmetric walls.

A special case of symmetric walls are neutral walls, i.e., $\mathcal{U}_{+}(z)=\mathcal{U}_{-}(z) \equiv 0$. In this case Eq. (45) reduces to

$$
n(z ; L)=\frac{n_{0}}{L}\left\{1+\pi\left(n_{0} L^{2}\right) C\left[\left(\frac{z}{L}\right)^{2}-\frac{1}{12}\right]+\mathcal{O}\left(n_{0} L^{2}\right)^{2}\right\} .
$$

In the case of hard spheres, $C=g\left(\sigma^{+}\right)$, up to order $\mathcal{O}\left(L^{2}\right)$ the density profile, Eq. (51), satisfies the contact theorem $n(z=$ $\pm L / 2)=p / k_{B} T$, see, e.g., Refs. 50 and 51 with the transversal pressure $p=k_{B} T n_{0} / L+k_{B} T A^{-1} \partial \ln Z /\left.\partial L\right|_{N, A}$ exerted on the walls, which has been evaluated recently ${ }^{43}$ to

$$
\begin{aligned}
p & =n_{0} k_{B} T / L\left[1+\frac{1}{6} \pi\left(n_{0} L^{2}\right) g\left(\sigma^{+}\right)+\mathcal{O}\left(L^{4}\right)\right] \\
& \equiv k_{B} \operatorname{Tn}(z= \pm L / 2 ; L) .
\end{aligned}
$$

\section{Correlation functions and their Fourier transforms}

The various two-point correlation functions introduced in Sec. II involve the 1-particle density $\rho^{(1)}(\vec{r} z ; L)$ from Eq. (44) and the 2-particle density $\rho^{(2)}\left(\vec{r} z, \vec{r}^{\prime} z^{\prime} ; L\right)$. From Eqs. (6) and (39) one obtains for $m=2$ the expansion

$$
\begin{aligned}
\rho^{(2)} & \left(\vec{r} z, \vec{r}^{\prime} z^{\prime} ; L\right) \\
= & \rho_{\perp}^{(1)}(z ; L) \rho_{\perp}^{(1)}\left(z^{\prime} ; L\right) \\
& \times\left\{\left[1+v_{1}^{\perp}\left(z, z^{\prime}\right) v_{1}^{\|}\left(\vec{r}, \vec{r}^{\prime}\right)\right] \rho_{\|}^{(2)}\left(\vec{r}, \vec{r}^{\prime}\right)\right. \\
& +\left[\left\langle v_{1}^{\perp}\left(z, z_{3}\right)\right\rangle_{\perp}+\left\langle v_{1}^{\perp}\left(z^{\prime}, z_{3}\right)\right\rangle_{\perp}\right] \int d^{2} r_{3} v_{1}^{\|}\left(\vec{r}, \vec{r}_{3}\right) \rho_{\|}^{(3)}\left(\vec{r}, \vec{r}^{\prime}, \vec{r}_{3}\right) \\
& +\frac{1}{2}\left\langle v_{1}^{\perp}\left(z_{3}, z_{4}\right)\right\rangle_{\perp} \int d^{2} r_{3} \int d^{2} r_{4} v_{1}^{\|}\left(\vec{r}_{3}, \vec{r}_{4}\right) \\
& \times\left[\rho_{\|}^{(4)}\left(\vec{r}, \vec{r}^{\prime}, \vec{r}_{3}, \vec{r}_{4}\right)-\rho_{\|}^{(2)}\left(\vec{r}, \vec{r}^{\prime}\right) \rho_{\|}^{(2)}\left(\vec{r}_{3}, \vec{r}_{4}\right)\right] \\
& -\left\langle v_{1}^{\perp}\left(z_{3}, z_{4}\right)\right\rangle_{\perp}\left(\frac{n_{0}^{2} \kappa_{T}}{2 \beta}\right)\left(\left.\frac{\partial}{\partial n_{0}}\right|_{T} \rho_{\|}^{(2)}\left(\vec{r}, \vec{r}^{\prime}\right)\right) \\
& \left.\times\left.\int d^{2} r_{4} v_{1}^{\|}\left(\vec{r}_{3}, \vec{r}_{4}\right) \frac{\partial}{\partial n_{0}}\right|_{T} \rho_{\|}^{(2)}\left(\vec{r}_{3}, \vec{r}_{4}\right)+\mathcal{O}\left(L^{4}\right)\right\}
\end{aligned}
$$

The leading order factorizes to $\rho_{\perp}^{(1)}(z ; L) \rho_{\perp}^{(1)}\left(z^{\prime} ; L\right)$ $\rho_{\|}^{(2)}\left(\vec{r}, \vec{r}^{\prime}\right)=n_{0}^{2} \rho_{\perp}^{(1)}(z ; L) \rho_{\perp}^{(1)}\left(z^{\prime} ; L\right) g\left(\left|\vec{r}-\vec{r}^{\prime}\right|\right)$, which is a consequence of the decoupling of the lateral and transversal degrees of freedom in the limit $L \rightarrow 0$. Note, that this expression is different from the superposition approximation originally suggested in Ref. 52, where $\rho^{(2)}\left(\vec{r} z, \vec{r}^{\prime} z^{\prime} ; L\right)$ is replaced by $\rho^{(1)}(z ; L) \rho^{(1)}\left(z^{\prime} ; L\right) g^{(3 D)}\left(\sqrt{\left(\vec{r}-\vec{r}^{\prime}\right)^{2}+\left(z-z^{\prime}\right)^{2}}\right)=$ $\rho^{(1)}(z ; L) \rho^{(1)}\left(z^{\prime} ; L\right)\left[g^{(3 D)}(|\vec{r}-\vec{r}|)+\mathcal{O}\left(L^{2}\right)\right]$ with $g^{(3 D)}(|\vec{x}|)$ being the pair-distribution function of the bulk fluid.

The first-order correction of $\rho^{(2)}\left(\vec{r} z, \vec{r}^{\prime} z^{\prime} ; L\right)$ requires $m$ particle densities $\rho_{\|}^{(m)}\left(\vec{r}_{1}, \ldots, \vec{r}_{m}\right)$ with $m=2,3$, and 4 of the $2 \mathrm{D}$ reference fluid. These correlation functions can be determined either by computer simulations or by suitable approximations. For instance, one can combine integral-equation theory to evaluate $\rho_{\|}^{(2)}\left(\vec{r}_{1}, \vec{r}_{2}\right)$ and then to obtain in superposition approximation $\rho_{\|}^{(m)}$ for $m=3$ and $m=4$. With Eqs. (44) and (53) the first-order correction $g_{1}\left(\vec{r} z, \vec{r}^{\prime} z^{\prime} ; L\right)$ of the pair-distribution function $g\left(\vec{r} z, \vec{r}^{\prime} z^{\prime} ; L\right)$ follows from Eq. (11). Using the factorization property of Eq. (41) one can readily prove that the correction vanishes $g_{1}\left(\vec{r} z, \vec{r}^{\prime} z^{\prime} ; L\right) \rightarrow 0$ for $\left|\vec{r}-\vec{r}^{\prime}\right| \rightarrow \infty$. The $\left(z, z^{\prime}\right)$-dependence of $g\left(\vec{r} z, \vec{r}^{\prime} z^{\prime} ; L\right)$ is given by $\left(z-z^{\prime}\right)^{2},\left\langle\left(z-z_{3}\right)^{2}\right\rangle_{\perp}$, and $\left\langle\left(z^{\prime}-z_{3}\right)^{2}\right\rangle_{\perp}$.

Finally we determine the rapidity of convergence of various correlation functions and their Fourier transforms to their respective $2 \mathrm{D}$ counterparts.

From Eqs. (6), (7) and (11), and (37) it follows with $\tilde{\rho}_{1}^{(m)}=\mathcal{O}\left(L^{2}\right)$ for all $m$,

$$
g\left(\vec{r} z, \vec{r}^{\prime} z^{\prime} ; L\right)=g(r)+\mathcal{O}\left(L^{2}\right),
$$

independent of the type of the wall potential. The same holds for the total correlation function

$$
h\left(\vec{r} z, \vec{r}^{\prime} z^{\prime} ; L\right)=h(r)+\mathcal{O}\left(L^{2}\right) .
$$


To discuss the direct correlation function $c\left(\vec{r}, z, z^{\prime} ; L\right)$ in confined geometry, we employ the inhomogeneous Ornstein-Zernike equation (16). We will prove that $\delta h\left(\vec{r}, z, z^{\prime} ; L\right)=h\left(\vec{r}, z, z^{\prime} ; L\right)-h(r)=\mathcal{O}\left(L^{2}\right) \quad$ implies $\delta c\left(r, z, z^{\prime} ; L\right)=c\left(\vec{r}, z, z^{\prime} ; L\right)-c(r)=\mathcal{O}\left(L^{2}\right)$. Inserting the leading order into Eq. (16) and using the sum rule $\int n(z ; L) \mathrm{d} z$ $=n_{0}$ one recovers the homogeneous $2 \mathrm{D}$ Ornstein-Zernike equation

$$
c(\vec{r})=h(\vec{r})-n_{0} \int \mathrm{d}^{2} r^{\prime \prime} c\left(\vec{r}-\vec{r}^{\prime \prime}\right) h\left(\vec{r}^{\prime \prime}-\vec{r}^{\prime}\right) .
$$

The next order then constitutes an integral equation for the first correction of the direct correlation function

$$
\begin{aligned}
& \delta c\left(r, z, z^{\prime} ; L\right)=\delta h\left(r, z, z^{\prime} ; L\right) \\
& -n_{0} \int \mathrm{d}^{2} r^{\prime \prime} \int \mathrm{d} \tilde{z}^{\prime \prime} \delta c\left(\vec{r}-\vec{r}^{\prime \prime}, z, z^{\prime \prime}=\tilde{z}^{\prime \prime} L ; L\right) h\left(\vec{r}^{\prime \prime}-\vec{r}^{\prime}\right) \\
& -n_{0} \int \mathrm{d}^{2} r^{\prime \prime} \int \mathrm{d} \tilde{z}^{\prime \prime} c\left(\vec{r}-\vec{r}^{\prime \prime}\right) \delta h\left(\vec{r}^{\prime \prime}-\vec{r}^{\prime}, z^{\prime \prime}=\tilde{z}^{\prime \prime} L, z^{\prime} ; L\right) .
\end{aligned}
$$

In particular to this order the density profile does not contribute and with $\delta h=\mathcal{O}\left(L^{2}\right)$ we conclude that $\delta c=\mathcal{O}\left(L^{2}\right)$, irrespective of the particle-wall interaction.

The self and distinct part of the density-density correlation function, $G^{(s)}$ and $G^{(d)}$ from Eqs. (13) and (14), respectively, involve $\rho_{\perp}^{(1)}$. For analytic wall potentials we obtain with Eq. (48)

$$
G^{(s)}\left(\vec{r}, z, z^{\prime} ; L\right)=\frac{1}{L}\left[1+\mathcal{O}\left(L^{\gamma}\right)\right] \delta(\vec{r}) \delta\left(z-z^{\prime}\right),
$$

and

$$
G^{(d)}\left(\vec{r}, z, z^{\prime} ; L\right)=\frac{1}{L^{2}}\left[G^{(d)}(\vec{r})+\mathcal{O}\left(L^{\gamma}\right)\right],
$$

i.e., the first-order correction of $G^{(s)}$ and $G^{(d)}$ depends on the wall type.

The Fourier transforms of all these correlation functions readily follow

$$
\begin{aligned}
& g_{\mu \nu}(q)=L^{2} g(q)\left[\delta_{\mu 0} \delta_{\nu 0}+\mathcal{O}\left(L^{2}\right)\right], \\
& h_{\mu \nu}(q)=L^{2} h(q)\left[\delta_{\mu 0} \delta_{\nu 0}+\mathcal{O}\left(L^{2}\right)\right], \\
& c_{\mu \nu}(q)=L^{2} c(q)\left[\delta_{\mu 0} \delta_{\nu 0}+\mathcal{O}\left(L^{2}\right)\right] .
\end{aligned}
$$

We find the leading order for $L \rightarrow 0$ is merely given by the 0 -0 component, while the correction and all remaining matrix elements vanish as $\mathcal{O}\left(L^{2}\right)$ irrespective of the wall potential. This stands in contrast to the corresponding Fourier transform of $G^{(s)}$ and $G^{(d)}$ :

$$
S_{\mu \nu}^{(s)}(q ; L)= \begin{cases}1 & \text { for } \mu=v, \\ \mathcal{O}\left(L^{\gamma}\right) & \text { else }\end{cases}
$$

$$
S_{\mu \nu}^{(d)}(q ; L)= \begin{cases}S^{(d)}(q)\left[1+\mathcal{O}\left(L^{2}\right)\right] & \text { for } \mu=v=0, \\ \mathcal{O}\left(L^{\gamma}\right) & \text { else, }\end{cases}
$$

which yields for the generalized structure factor

$$
S_{\mu \nu}(q ; L)= \begin{cases}S(q)\left[1+\mathcal{O}\left(L^{2}\right)\right] & \text { for } \mu=v=0, \\ \left(1-\delta_{\mu 0}\right) \delta_{\mu \nu}+\mathcal{O}\left(L^{\gamma}\right) & \text { else, }\end{cases}
$$

where $S(q)$ refers to the static structure factor of the 2D reference fluid. $S_{\mu \nu}^{(s)}(q ; L), S_{\mu \nu}^{(d)}(q ; L)$, and $S_{\mu \nu}(q ; L)$ have a proper 2D limit only if the criterion (cf. Eq. (28)) is fulfilled. In case of proper convergence these structure factors become diagonal in $\mu$ and $\nu$. By contrast, since $S_{00}^{(d)}(q ; L)$ $=\int \mathrm{d}^{2} r \mathrm{~d} z \mathrm{~d} z^{\prime} n(z)\left[h(r)+\mathcal{O}\left(L^{2}\right)\right] n\left(z^{\prime}\right) \mathrm{e}^{-i \vec{q} \cdot \vec{r}} / n_{0}=n_{0} h(q)[1$ $\left.+\mathcal{O}\left(L^{2}\right)\right]$ due to the normalization $\int \mathrm{d} z n(z ; L)=n_{0}$, the convergence of the in-planar structure factor $S_{00}(q)$ is of order $\mathcal{O}\left(L^{2}\right)$ and irrespective of the particle-wall interaction.

Finally we mention that the analytic dependence of $\mathcal{U}(z ; L)$ on $z$ is too strict. It is sufficient to require $\mathcal{U}(z$ $=\tilde{z} L ; L)-\mathcal{U}(0 ; L)=o\left(L^{0}\right)$, where the little- $o$ Landau symbol $h(x)=o(x)$ indicates that for $x \rightarrow 0$ the function $h(x)$ converges faster to zero than $x$. In this case we have to replace $\mathcal{O}(L)$ by $o\left(L^{0}\right)$ in the estimates and find that the static quantities converge to their respective 2D limit, yet without specification on the rapidity of convergence.

\section{SUMMARY AND CONCLUSIONS}

For a fluid confined between two parallel walls we have investigated the regime of quasi-two-dimensionality, where the accessible distance $L$ between the adjacent walls becomes small. The focus has been on the behavior of structural quantities, which are entirely described by the $m$-particle density. This quantity can be factorized into a density of transversal dof depending only on the wall-potential and into a reduced $m$-particle density containing the mutual interactions of the particles in the slit. In the limit $L \rightarrow 0$ we analytically determine the $m$-particle density by taking the transversal degrees of freedom as a small perturbation.

To leading order the reduced $m$-particle density is identical to the corresponding $m$-particle density of the $2 \mathrm{D}$ reference fluid, which is a consequence of the decoupling mechanism of the transversal and lateral dof in the 2D limit. The next-to-leading order contains information on the transversal degrees of freedom $\left(z_{1}, \ldots, z_{m}\right)$, which we find to be quadratic in $z_{i}$ and $z_{j}$. Therefore, the leading corrections are of order $L^{2}$ irrespective of the particular particle-wall interactions. Our analysis has been made explicit for smooth interaction potentials, but we also outline the strategy in the case of hard-core interactions, where a systematic cluster expansion is inevitable. ${ }^{43}$

The most basic structural entity characterizing the structure of the liquid is the density profile, which merely depends on the transversal position $z$ due to translational symmetry along the walls. Its first-order correction is proportional to $-\beta \int \mathrm{d} r \mathcal{V}^{\prime}(r) g(r)$ (cf. Eq. (44)) with $g(r)$ being the pair-distribution function of the $2 \mathrm{D}$ reference fluid. This factor represents a measure of the profile's curvature and therefore of the deviation from flatness. In case of a hard-sphere 
fluid this proportionality factor becomes $g\left(\sigma^{+}\right)$, the 2D pairdistribution function at contact. This finding differs from the result in Ref. 21 for the curvature of the density profile. There, the first equation of the Born-Green-Yvon hierarchy for the 2-particle density has been truncated via the superposition approximation. ${ }^{52}$ This superposition approximation involves the pair-distribution function of the 3D bulk fluid instead of $g\left(\sigma^{+}\right)$causing a substantial quantitative mismatch for the curvature. Our result proves, that for $L \rightarrow 0$ the superposition principle becomes exact only if the $3 \mathrm{D}$ pair-distribution function is replaced by its $2 \mathrm{D}$ counterpart.

For the various structural quantities we uncover a hierarchy in terms of the rapidity of convergence. For example, the pair-distribution function $g\left(\vec{r}, z, z^{\prime}\right)$, which is closely related to the 2-particle density (cf. Eq. (11)) converges as $L^{2}$ and its 2D limit always exists. Similarly, this holds for the total and direct correlation function and their rapidity of convergence is independent of the specific type of wall potential. By contrast, for different structural entities we have found that the $2 \mathrm{D}$ limit is rather subtle and depends sensitively on the properties of the wall potentials $\mathcal{U}_{ \pm}(z)$. For instance, for the density profile we have demonstrated that for wall potentials diverging for $z$ $\rightarrow 0$, such as for the Lennard-Jones or Coulomb potential, the profile becomes singular for $L \rightarrow 0$. The same holds for the density-density correlation function $G\left(\vec{r} z, \vec{r}^{\prime} z^{\prime} ; L\right)$. For such wall potentials these quantities do not become flat in the $2 \mathrm{D}$ limit. To obtain a proper $2 \mathrm{D}$ limit the wall potential $\mathcal{U}(z ; L)$ has to fulfill a smoothness criterion as discussed in Subsection III A. It is fulfilled if $\mathcal{U}_{ \pm}(z)$ is analytic at $z=0$. However, the condition of analyticity can be weakened. Convergence also holds provided the wall potential approaches its average value everywhere in the slit.

In both cases, only the zero mode in the Fourier decomposition of the density profile survives and coincides with the planar density $n_{0}$. Similarly, the non-diagonal elements of the structure factors $S_{\mu \nu}(q ; L)$ vanish as the walls approach each other, whereas $S_{00}(q ; L)$ converges to $S(q)$, the structure factor of the 2D fluid, and the remaining diagonal elements become unity. While generally a proper convergence of the structure factor depends on the particle-wall interactions, we find that $S_{00}(q ; L)$ always converges as $\mathcal{O}\left(L^{2}\right)$ to its $2 \mathrm{D}$ counterpart irrespective of the wall potential, which is a manifestation of its sole dependence on the lateral coordinates of the fluid. ${ }^{43}$ In the case of the direct correlation function, we find that the leading order is determined by the matrix element $c_{00}(q ; L)$, which is related to the $2 \mathrm{D}$ direct correlation function $c(q)$ for $L \rightarrow 0$ (cf. Eq. (62)). The leading correction and all remaining Fourier components $c_{\mu \nu}(q ; L)$ converge as $\mathcal{O}\left(L^{2}\right)$ independent of the particle-wall interaction.

Our results provide estimates on the rapidity of convergence. However, the range of validity of the leading order remains unknown in general. In the case of the equilibrium phase diagram for hard spheres of diameter $\sigma$ and neutral walls it has been shown that for $L \lesssim 0.5 \sigma$ the leading order describes the phase transition lines quantitatively (cf. Figure 3 in Ref. 43), i.e., there is almost no influence of the transversal degrees of freedom up to $L \lesssim 0.5 \sigma$. We expect the density profile and the density-density correlation function to be described quantitatively for similar plate distances. Let us compare our predictions with the Monte Carlo results of Ref. 21 for the same system with fixed chemical potential corresponding to a bulk density $n \sigma^{3}=0.5$. Their Figure 1 shows that the profile is practically flat for the smallest wall separation $L=0.1 \sigma$. Consequently, the confined fluid behaves approximately as a $2 \mathrm{D}$ fluid. However, for the next largest value $L=0.5 \sigma$ presented in the same figure the profile is already parabolic and the subsequent figure of that paper reveals that the parabolic shape extends up to $L=0.8 \sigma$. Hence, the leading-order correction in $n_{0} L^{2}$ in Eq. (51) is sufficient to describe the density profile up to $L=0.8 \sigma$, at least for the chemical potential chosen to match the bulk density $n \sigma^{3}=0.5$.

The analytical results including leading-order corrections elaborated in this work provide testable predictions, which can be rationalized by computer simulations or experiments. As our results become exact in the limit of small wall separations, they serve as a reference for approximate theories. For instance, in case of hard spheres enclosed between neutral walls we have proven that the curvature of the density profile is determined by the 2D contact value $g\left(\sigma^{+}\right)$rather than by $g^{(3 D)}\left(\sigma^{+}\right)$, as it has been suggested by the superposition approximation. ${ }^{21}$ Vice versa, our finding enables to measure the contact value of the $2 \mathrm{D}$ pair-distribution function via the curvature of the parabolic profile.

\section{ACKNOWLEDGMENTS}

We kindly thank Bob Evans for stimulating discussions. This work has been supported by the Deutsche Forschungsgemeinschaft DFG via the Research Unit FOR1394 "Nonlinear response to probe vitrification." S.L. acknowledges support by the Cluster of Excellence "Engineering of Advanced Materials" at the University of Erlangen-Nuremberg funded by the DFG.

\section{APPENDIX A: CALCULATION OF $\tilde{\rho}_{l}^{(m)}\left(\vec{r}_{1} z_{1}, \ldots, \vec{r}_{m} z_{m}, L\right)$ FOR $I=0,1$}

We introduce in this Appendix a shorthand notation, where the positions $\vec{x}_{j}=\left(\vec{r}_{j}, z_{j}\right)$ are abbreviated by $\left(j^{\|}, j^{\perp}\right)$ $=j$ and we adopt the convention

$$
\begin{aligned}
\int d j(\cdots) & \equiv \int d^{\|} j \int d^{\perp} j(\cdots) \\
& \equiv \int_{A} d^{2} r_{j} \int_{-L / 2}^{L / 2} d z_{j} \exp \left[-\beta \mathcal{U}\left(z_{j} ; L\right)\right](\cdots) / z_{\perp}(L) .
\end{aligned}
$$

Here, $z_{\perp}(L)=\int_{-L / 2}^{L / 2} d z \exp [-\beta \mathcal{U}(z ; L)]$ refers to the configurational part of the partition function for a single particle interacting with the walls only. Functions $f^{\|}\left(\vec{r}_{1}, \vec{r}_{2}, \ldots\right)$ and $f^{\perp}\left(z_{1}\right.$, $z_{2}, \ldots$ ) depending on the lateral and transversal dof, respectively, are denoted by $f^{\|}(1,2, \ldots)$ and $f^{\perp}(1,2, \ldots)$, so that the superscripts uniquely define the variables. 
Substituting Eq. (34) into the integral term of Eq. (8) and using the decomposition of the interaction potential

$$
\begin{aligned}
\sum_{1 \leq i<j \leq N} v_{1}(i, j)= & \sum_{1 \leq i<j \leq m} v_{1}(i, j)+\sum_{i=1}^{m} \sum_{j=m+1}^{N} v_{1}(i, j) \\
& +\sum_{m+1 \leq i<j \leq N} v_{1}(i, j)
\end{aligned}
$$

one obtains

$$
\begin{aligned}
& \frac{N !}{(N-m) !} \frac{1}{Z_{\|}} \int \prod_{j=m+1}^{N} d j \exp \left[-\beta V_{0}(1, \ldots, N)\right] \\
& =\frac{N !}{(N-m) !} \frac{1}{Z_{\|}} \int \prod_{j=m+1}^{N} d j \exp \left[-\beta V_{0}^{\|}(1, \ldots, N)\right] \\
& \quad \times\left\{1+\sum_{1 \leq i<j \leq m} v_{1}(i, j)+(N-m) \sum_{i=1}^{m} v_{1}(i, m+1)\right. \\
& \left.\quad+\frac{1}{2}(N-m)(N-m-1) v_{1}(m+1, m+2)+\mathcal{O}\left(L^{4}\right)\right\},
\end{aligned}
$$

where the invariance under relabeling of the summation indices has been employed. The rhs of Eq. (A3) can be expressed by the $m$-particle density of the $2 \mathrm{D}$ reference fluid

$$
\begin{aligned}
& \rho_{\|}^{(m)}(1, \ldots, m ; N) \\
& \quad:=\frac{N !}{(N-m) !} \int \prod_{j=m+1}^{N} d^{\|} j \exp \left[-\beta V_{0}^{\|}(1, \ldots, N)\right] / Z_{\|},
\end{aligned}
$$

which then yields

$$
\begin{aligned}
& \frac{N !}{(N-m) !} \frac{1}{Z_{\|}} \int \prod_{j=m+1}^{N} d j \exp \left[-\beta V_{0}(1, \ldots, N)\right] \\
& =\rho_{\|}^{(m)}(1, \ldots, m ; N)+\sum_{1 \leq i<j \leq m} v_{1}(i, j) \rho_{\|}^{(m)}(1, \ldots, m ; N) \\
& \quad+\sum_{i=1}^{m} \int d(m+1) v_{1}(i, m+1) \rho_{\|}^{(m+1)}(1, \ldots, m+1 ; N) \\
& \quad+\frac{1}{2} \int d(m+1) \int d(m+2) v_{1}(m+1, m+2) \\
& \quad \times \rho_{\|}^{(m+2)}(1, \ldots, m+2 ; N)+\mathcal{O}\left(L^{4}\right) .
\end{aligned}
$$

Here, the variable $N$ of $\rho_{\|}^{(m)}(1, \ldots, m ; N)$ shall indicate, that the support of the $m$-particle densities is restricted only to the finite system of area $A$ with finite particle number $N$. The partition function $Z(L)$ follows similarly, but does not require the decomposition of Eq. (A2). Taking $\int d^{\perp} j$ $=\int d z_{j} \exp \left[-\beta \mathcal{U}\left(z_{j} ; L\right)\right] / z_{\perp}(L)=1$ into account one infers with Eq. (34) and $z_{\perp}^{N}(L)=Z_{\perp}(L)$ :

$Z(L)$

$$
\begin{aligned}
= & Z_{\perp}(L) \int \prod_{j=1}^{N} d j \exp \left[-\beta V_{0}(1, \ldots, N)\right] \\
= & Z_{\perp}(L) \int \prod_{j=1}^{N} d j \exp \left[-\beta V_{0}^{\|}(1, \ldots, N)\right] \\
& \times\left[1+\frac{1}{2} \frac{N !}{(N-2) !} v_{1}(1,2)+\mathcal{O}\left(L^{4}\right)\right] \\
= & Z_{\perp}(L) Z_{\|}\left[1+\frac{1}{2} \int d 1 \int d 2 v_{1}(1,2) \rho_{\|}^{(2)}(1,2 ; N)+\mathcal{O}\left(L^{4}\right)\right] .
\end{aligned}
$$

Then the normalization factor (Eq. (9)) becomes

$$
\mathcal{N}(L)=1-\frac{1}{2} \int d 1 \int d 2 v_{1}(1,2) \rho_{\|}^{(2)}(1,2 ; N)+\mathcal{O}\left(L^{4}\right)
$$

Substituting Eqs. (A5) and (A7) into Eq. (8) and renaming the integration variables in Eq. (A7), we arrive at

$$
\begin{aligned}
\tilde{\rho}^{(m)}(1, \ldots, m ; L) & \\
= & {\left[1+\sum_{1 \leq i<j \leq m} v_{1}(i, j)\right] \rho_{\|}^{(m)}(1, \ldots, m ; N) } \\
& +\sum_{i=1}^{m} \int d(m+1) v_{1}(i, m+1) \rho_{\|}^{(m+1)}(1, \ldots, m+1 ; N) \\
& +\frac{1}{2} \int d(m+1) \int d(m+2) v_{1}(m+1, m+2) \\
& \times\left[\rho_{\|}^{(m+2)}(1, \ldots, m+2 ; N)\right. \\
& \left.-\rho_{\|}^{(m)}(1, \ldots, m ; N) \rho_{\|}^{(2)}(m+1, m+2 ; N)\right] \\
& +\mathcal{O}\left(L^{4}\right) .
\end{aligned}
$$

Comparison with Eq. (37) leads to

$$
\tilde{\rho}_{0}^{(m)}(1, \ldots, m ; N ; L)=\rho_{\|}^{(m)}(1, \ldots, m ; N),
$$

and

$$
\begin{aligned}
\tilde{\rho}_{1}^{(m)}(1, \ldots, m ; N ; L) & \\
= & \sum_{1 \leq i<j \leq m} v_{1}(i, j) \rho_{\|}^{(m)}(1, \ldots, m ; N) \\
& +\sum_{i=1}^{m} \int d(m+1) v_{1}(i, m+1) \rho_{\|}^{(m+1)}(1, \ldots, m+1 ; N) \\
& +\frac{1}{2} \int \mathrm{d}(m+1) \int \mathrm{d}(m+2) v_{1}(m+1, m+2) \\
& \times\left[\rho_{\|}^{(m+2)}(1, \ldots, m+2 ; N)\right. \\
& \left.-\rho_{\|}^{(m)}(1, \ldots, m ; N) \rho_{\|}^{(2)}(m+1, m+2 ; N)\right] .
\end{aligned}
$$


The first-order correction for $m=2$, Eq. (A10), has the same structure as the correction obtained for a bulk fluid perturbed by a pair potential, see Ref. 53. It has been pointed out in Ref. 47 that this result is formal, i.e., valid for the finite system only. To obtain its TDlimit we use a relation connecting the $m$-particle density of the finite system $\rho_{\|}^{(m)}(1, \ldots, m ; N)$ to the corresponding quantity $\rho_{\|}^{(m)}(1, \ldots, m ; \infty) \equiv \rho_{\|}^{(m)}(1, \ldots, m)$ of the infinite system: ${ }^{49}$

$$
\begin{aligned}
\rho_{\|}^{(m)} & (1, \ldots, m ; N) \\
= & \rho_{\|}^{(m)}(1, \ldots, m)-\left.\frac{1}{N}\left(\frac{n_{0} \kappa_{T}}{2 \beta}\right) n_{0}^{2} \frac{\partial^{2}}{\partial n_{0}^{2}}\right|_{T} \rho_{\|}^{(m)}(1, \ldots, m) \\
& +o\left(N^{-1}\right) .
\end{aligned}
$$

Here, $\kappa_{T}=\left[n_{0} \partial \Sigma /\left.\partial n_{0}\right|_{T}\right]^{-1}$ refers to the 2D isothermal compressibility of the reference fluid with surface tension given by $\Sigma=n_{0} k_{B} T+k_{B} T \partial \ln \left(Z_{\|}\right) /\left.\partial A\right|_{T, N}$.

The second term in Eq. (A11) is of order $1 / N$, however it gives rise to an intensive contribution via the double integral of Eq. (A10). Its generic contribution stems from such $\left((m+1)^{\|},(m+2)^{\|}\right)$with a distance to the fixed positions $\left(1^{\|}, \ldots, m^{\|}\right)$, which is much larger than the correlation length. In this case one can use in Eq. (A11) the factorization property

$$
\rho_{\|}^{(m+2)}(1, \ldots, m+2) \rightarrow \rho_{\|}^{(m)}(1, \ldots, m) \rho_{\|}^{(2)}(m+1, m+2),
$$

see Ref. 49. As a final result, we obtain

$$
\begin{aligned}
& \tilde{\rho}_{1}^{(m)}(1, \ldots, m ; L) \\
& =\sum_{1 \leq i<j \leq m} v_{1}(i, j) \rho_{\|}^{(m)}(1, \ldots, m) \\
& \quad+\sum_{i=1}^{m} \int d(m+1) v_{1}(i, m+1) \rho_{\|}^{(m+1)}(1, \ldots, m+1) \\
& \quad+\frac{1}{2} \int \mathrm{d}(m+1) \int \mathrm{d}(m+2) v_{1}(m+1, m+2) \\
& \quad \times\left[\rho_{\|}^{(m+2)}(1, \ldots, m+2)-\rho_{\|}^{(m)}(1, \ldots, m) \rho_{\|}^{(2)}(m+1, m+2)\right] \\
& \quad-\left(\frac{n_{0}^{2} \kappa_{T}}{2 \beta}\right)\left(\left.\frac{\partial}{\partial n_{0}}\right|_{T} \rho_{\|}^{(m)}(1, \ldots, m)\right) \int \mathrm{d}(m+2) \\
& \quad \times\left. v_{1}(m+1, m+2) \frac{\partial}{\partial n_{0}}\right|_{T} \rho_{\|}^{(2)}(m+1, m+2) .
\end{aligned}
$$

For the last term we have used that the integrand of the double integral, Eq. (A11), depends on $\left|(m+2)^{\|}-(m+1)^{\|}\right|$ by translational invariance yielding an intensive term proportional to $A / N=1 / n_{0}$.

\section{APPENDIX B: CALCULATION OF THE REDUCED 1-PARTICLE DENSITY $\tilde{\rho}_{1}^{(1)}\left(\vec{r}_{1} z_{1} ; L\right)$}

We employ the shorthand notation as introduced in Appendix A to evaluate Eq. (39) in the case of $m=1$ :

$$
\begin{aligned}
\tilde{\rho}_{1}^{(1)}(1 ; L) \\
=\left\langle v_{1}^{\perp}(1,2)\right\rangle_{\perp} \int d^{\|} 2 v_{1}^{\|}(1,2) \rho_{\|}^{(2)}(1,2) \\
+\frac{1}{2}\left\langle v_{1}^{\perp}(2,3)\right\rangle_{\perp} \int d^{\|} 2 \int d^{\|} 3 v_{1}^{\|}(2,3) \\
\quad \times\left[\rho_{\|}^{(3)}(1,2,3)-\rho_{\|}^{(1)}(1) \rho_{\|}^{(2)}(2,3)\right] \\
-\left.\left\langle v_{1}^{\perp}(2,3)\right\rangle_{\perp}\left(\frac{n_{0}^{2} \kappa_{T}}{2 \beta}\right) \int d^{\|} 3 v_{1}^{\|}(2,3) \frac{\partial}{\partial n_{0}}\right|_{T} \rho_{\|}^{(2)}(2,3),
\end{aligned}
$$

where $\left.\frac{\partial}{\partial n_{0}}\right|_{T} \rho_{\|}^{(1)}(1) \equiv 1$ has been used, since $\rho_{\|}^{(1)}(1) \equiv n_{0}$. Substituting $\left.\frac{\partial}{\partial n_{0}}\right|_{T} \rho_{\|}^{(2)}(2,3)$ from Eq. (40) into Eq. (B1) yields for the last term of Eq. (B1):

$$
\begin{aligned}
- & \left\langle v_{1}^{\perp}(2,3)\right\rangle_{\perp}\left\{\int d^{\|} 3 v_{1}^{\|}(2,3) \rho_{\|}^{(2)}(2,3)\right. \\
& \left.+\frac{1}{2} \int d^{\|} 3 v_{1}^{\|}(2,3) \int d^{\|} 4\left[\rho_{\|}^{(3)}(2,3,4)-\rho_{\|}^{(2)}(2,3) \rho_{\|}^{(1)}(4)\right]\right\} .
\end{aligned}
$$

Consequently we obtain after renaming dummy variables:

$$
\begin{aligned}
\tilde{\rho}_{1}^{(1)}(1 ; L) \\
=\left[\left\langle v_{1}^{\perp}(1,2)\right\rangle_{\perp}-\left\langle v_{1}^{\perp}(2,3)\right\rangle_{\perp}\right] \int d^{\|} 2 v_{1}^{\|}(1,2) \rho_{\|}^{(2)}(1,2) \\
\quad+\frac{1}{2}\left\langle v_{1}^{\perp}(2,3)\right\rangle_{\perp}\left\{\int d^{\|} 2 d^{\|} 3 v_{1}^{\|}(2,3)\right. \\
\quad \times\left[\rho_{\|}^{(3)}(1,2,3)-\rho_{\|}^{(1)}(1) \rho_{\|}^{(2)}(2,3)\right] \\
\left.\quad-\int d^{\|} 3 d^{\|} 4 v_{1}^{\|}(2,3)\left[\rho_{\|}^{(3)}(2,3,4)-\rho_{\|}^{(1)}(4) \rho_{\|}^{(2)}(2,3)\right]\right\} .
\end{aligned}
$$

By translational invariance, the first term of the curly bracket in Eq. (B3) does not depend on $1^{\|} \cong \vec{r}_{1}$ and the second term is independent of $2^{\|} \cong \vec{r}_{2}$. After renaming the label $4 \rightarrow 1$ and employing the permutation invariance of $\rho_{\|}^{(3)}(1,2,3)$, one infers

$$
\begin{aligned}
\lim _{A \rightarrow \infty} & {\left[\frac{1}{A} \int d^{\|} 1 \int d^{\|} 2 \int d^{\|} 3 v_{1}^{\|}(2,3)\right.} \\
& \times\left(\rho_{\|}^{(3)}(1,2,3)-\rho_{\|}^{(1)}(1) \rho_{\|}^{(2)}(2,3)\right) \\
- & \frac{1}{A} \int d^{\|} 2 \int d^{\|} 3 \int d^{\|} 4 v_{1}^{\|}(2,3)\left(\rho_{\|}^{(3)}(2,3,4)\right. \\
- & \left.\left.\rho_{\|}^{(1)}(4) \rho_{\|}^{(2)}(2,3)\right)\right]=0 .
\end{aligned}
$$


Finally, with Eq. (B4) it follows

$$
\begin{aligned}
\tilde{\rho}_{1}^{(1)}(1 ; L)= & {\left[\left\langle v_{1}^{\perp}(1,2)\right\rangle_{\perp}-\left\langle v_{1}^{\perp}(2,3)\right\rangle_{\perp}\right] } \\
& \times \int d^{\|} 2 v_{1}^{\|}(1,2) \rho_{\|}^{(2)}(1,2),
\end{aligned}
$$

which is independent of $1^{\|} \cong \vec{r}_{1}$.

\section{APPENDIX C: CANONICAL AVERAGES OF THE CLUSTER FUNCTION OF HARD SPHERES}

In the case of hard-sphere interaction the first-order correction $\tilde{\rho}_{1}^{(m)}$ contains integrals of the subsequent form

$$
\begin{aligned}
& I_{1}^{(m)}\left(z_{1}, \vec{r}_{1}, \ldots, \vec{r}_{m} ; L\right) \\
& =\int d z_{m+1} \rho_{\perp}^{(1)}\left(z_{m+1}\right) \int d^{2} r_{m+1} f\left(\vec{r}_{1} z_{1}, \vec{r}_{m+1} z_{m+1}\right) \\
& \quad \times \rho_{\|}^{(m+1)}\left(\vec{r}_{1}, \ldots, \vec{r}_{m+1} ; \sigma_{L}\right)
\end{aligned}
$$

where $\rho_{\|}^{(m)}\left(\vec{r}_{1}, \ldots, \vec{r}_{m} ; \sigma_{L}\right)$ refers to the 2D $m$-particle density of hard disks with reduced diameter $\sigma_{L}$. Inserting for $f\left(\vec{r}_{1} z_{1}, \vec{r}_{m+1} z_{m+1}\right)$ the explicit representation of the clusterfunction from Eq. (42) and using $\vec{r}_{m+1}=\vec{r}_{1}+\vec{r}_{1, m+1}$, one obtains

$$
\begin{aligned}
I_{1}^{(m)}\left(z_{1}, \vec{r}_{1}, \ldots, \vec{r}_{m} ; L\right) \\
=\int d z_{m+1} \rho_{\perp}^{(1)}\left(z_{m+1}\right) \int_{0}^{2 \pi} d \varphi_{m+1} \int_{0}^{\infty} d r_{1, m+1} r_{1, m+1} \\
\quad \times\left[\Theta\left(r_{1, m+1}^{2}+\left(z_{1}-z_{m+1}\right)^{2}-\sigma^{2}\right)-\Theta\left(r_{1, m+1}^{2}-\sigma_{L}^{2}\right)\right] \\
\quad \times \rho_{\|}^{(m+1)}\left(\vec{r}_{1}, \ldots, \vec{r}_{1}+r_{1, m+1} \vec{e}\left(\varphi_{1, m+1}\right) ; \sigma_{L}\right) \\
=-\int d z_{m+1} \rho_{\perp}^{(1)}\left(z_{m+1}\right) \int_{0}^{2 \pi} d \varphi_{m+1} \int_{\sigma_{L}}^{\sqrt{\sigma^{2}-\left(z_{1}-z_{m+1}\right)^{2}}} d r_{1, m+1} \\
\quad \times r_{1, m+1} \rho_{\|}^{(m+1)}\left(\vec{r}_{1}, \ldots, \vec{r}_{1}+r_{1, m+1} \vec{e}\left(\varphi_{1, m+1}\right) ; \sigma_{L}\right),
\end{aligned}
$$

where the unit vector $\vec{e}\left(\varphi_{1, m+1}\right)=\vec{r}_{1, m+1} / r_{1, m+1}$ has been introduced. The $r_{1, m+1}$-integration interval converges to zero for $L \rightarrow 0$. Therefore, one can expand the integral around its lower bound

$$
\begin{aligned}
& I_{1}^{(m)}\left(z_{1}, \vec{r}_{1}, \ldots, \vec{r}_{m} ; L\right) \\
& =-\frac{1}{2} \int d z_{m+1} \rho_{\perp}^{(1)}\left(z_{m+1}\right)\left[\sigma^{2}-\left(z_{1}-z_{m+1}\right)^{2}-\sigma_{L}^{2}\right] \\
& \quad \times \int_{0}^{2 \pi} d \varphi_{m+1} \rho_{\|}^{(m+1)}\left(\vec{r}_{1}, \ldots, \vec{r}_{1}+\sigma_{L} \vec{e}\left(\varphi_{m+1}\right) ; \sigma_{L}\right)+\mathcal{O}\left(L^{4}\right),
\end{aligned}
$$

where $\varphi_{1, m+1}=\varphi_{m+1}$ without restricting generality. With $\sigma_{L}=\sqrt{\sigma^{2}-L^{2}}$, one finally obtains

$$
\begin{aligned}
I_{1}^{(m)}\left(z_{1}, \vec{r}_{1}, \ldots, \vec{r}_{m} ; L\right) & \\
= & \pi\left[z_{1}^{2}-2 z_{1}\left\langle z_{m+1}\right\rangle_{\perp}+\left\langle z_{m+1}^{2}\right\rangle_{\perp}-L^{2}\right] \\
& \times\left\langle\rho_{\|}^{(m+1)}\left(\vec{r}_{1}, \ldots, \vec{r}_{1}+\sigma_{L} \vec{e}\left(\varphi_{m+1}\right) ; \sigma_{L}\right)\right\rangle_{\varphi} \\
& +\mathcal{O}\left(L^{4}\right),
\end{aligned}
$$

where angular averages are indicated by

$$
\langle(\cdots)\rangle_{\varphi}=\left(\frac{1}{2 \pi}\right) \int_{0}^{2 \pi} \mathrm{d} \varphi(\cdots) .
$$

The square bracket in Eq. (C4) corresponds to the first three terms occurring in Eq. (45), which arises from $\left\langle v_{1}^{\perp}\left(z_{1}, z_{2}\right)\right\rangle_{\perp}$ except for the term $L^{2}$ which stems from the diameter $\sigma_{L}$ of the reference fluid.

${ }^{1}$ C. Alba-Simionesco, B. Coasne, G. Dosseh, G. Dudziak, K. E. Gubbins, R. Radhakrishnan, and M. Sliwinska-Bartkowiak, J. Phys.: Condens. Matter 18, R15 (2006).

${ }^{2}$ S. Dietrich and A. Haase, Phys. Rep. 260, 1 (1995).

${ }^{3}$ H. Löwen, J. Phys.: Condens. Matter 13, R415 (2001).

${ }^{4}$ H. Löwen, J. Phys.: Condens. Matter 21, 474203 (2009).

${ }^{5}$ R. Evans, J. Phys.: Condens. Matter 2, 8989 (1990).

${ }^{6}$ K. Nygård, R. Kjellander, S. Sarman, S. Chodankar, E. Perret, J. Buitenhuis, and J. F. van der Veen, Phys. Rev. Lett. 108, 037802 (2012)

${ }^{7}$ E. C. Oğuz, A. Reinmüller, H. J. Schöpe, T. Palberg, R. Messina, and H. Löwen, J. Phys.: Condens. Matter 24, 464123 (2012).

${ }^{8}$ J. W. Zwanikken and M. O. de la Cruz, Proc. Natl. Acad. Sci. U.S.A. 110, 5301 (2013).

${ }^{9}$ K. Nygård, S. Sarman, and R. Kjellander, J. Chem. Phys. 139, 164701 (2013).

${ }^{10} \mathrm{~K}$. Binder, Thin Solid Films 20, 367 (1974).

${ }^{11}$ M. E. Fisher and H. Nakanishi, J. Chem. Phys. 75, 5857 (1981).

${ }^{12}$ R. L. C. Vink, K. Binder, and J. Horbach, Phys. Rev. E 73, 056118 (2006).

${ }^{13}$ Y. Liu, A. Z. Panagiotopoulos, and P. G. Debenedetti, J. Chem. Phys. 132, 144107 (2010).

${ }^{14}$ J. Klafter and J. M. Drake, Molecular Dynamics in Restricted Geometry (Wiley, New York, 1989).

${ }^{15}$ J. Mittal, T. M. Truskett, J. R. Errington, and G. Hummer, Phys. Rev. Lett. 100, 145901 (2008).

${ }^{16}$ C. R. Nugent, K. V. Edmond, H. N. Patel, and E. R. Weeks, Phys. Rev. Lett. 99, 025702 (2007).

${ }^{17}$ S. Lang, V. Boţan, M. Oettel, D. Hajnal, T. Franosch, and R. Schilling, Phys. Rev. Lett. 105, 125701 (2010).

${ }^{18}$ P. Gallo, M. Rovere, and E. Spohr, Phys. Rev. Lett. 85, 4317 (2000).

${ }^{19}$ S. H. Krishnan and K. G. Ayappa, Phys. Rev. E 86, 011504 (2012).

${ }^{20} \mathrm{~K}$. Watanabe, T. Kawasaki, and H. Tanaka, Nature Mater. 10, 512 (2011).

${ }^{21}$ V. Antonchenko, V. Ilyin, N. Makovsky, A. Pavlov, and V. Sokhan, Mol. Phys. 52, 345 (1984).

${ }^{22}$ J. Alejandre, M. Lozada-Cassou, and L. Degrève, Mol. Phys. 88, 1317 (1996).

${ }^{23}$ M. Schmidt and H. Löwen, Phys. Rev. Lett. 76, 4552 (1996).

${ }^{24}$ M. Schmidt and H. Löwen, Phys. Rev. E 55, 7228 (1997).

${ }^{25}$ A. Fortini and M. Dijkstra, J. Phys.: Condens. Matter 18, L371 (2006).

${ }^{26}$ N. Gribova, A. Arnold, T. Schilling, and C. Holm, J. Chem. Phys. 135, 054514 (2011)

${ }^{27}$ W. Qi, A. Gantapara, and M. Dijkstra, preprint arXiv:1307.1311 (2013).

${ }^{28}$ J. Henderson, Mol. Phys. 59, 89 (1986).

${ }^{29}$ P. Adams, J. R. Henderson, and J. P. R. B. Walton, J. Chem. Phys. 91, 7173 (1989).

${ }^{30}$ D. Henderson, S. Sokolowski, and D. Wasan, J. Stat. Phys. 89, 233 (1997).

${ }^{31}$ X. Xu and S. A. Rice, Proc. R. Soc. London A 464, 65 (2008).

${ }^{32}$ X. Xu and S. A. Rice, Phys. Rev. E 78, 011602 (2008).

${ }^{33}$ P. Tarazona, U. M. B. Marconi, and R. Evans, Mol. Phys. 60, 573 (1987).

${ }^{34}$ Y. Rosenfeld, M. Schmidt, H. Löwen, and P. Tarazona, J. Phys.: Condens. Matter 8, L577 (1996).

${ }^{35}$ Y. Rosenfeld, M. Schmidt, H. Löwen, and P. Tarazona, Phys. Rev. E 55, 4245 (1997).

${ }^{36}$ B. Götzelmann and S. Dietrich, Phys. Rev. E 55, 2993 (1997).

${ }^{37}$ K. W. Desmond and E. R. Weeks, Phys. Rev. E 80, 051305 (2009). 
${ }^{38}$ S. Neser, C. Bechinger, P. Leiderer, and T. Palberg, Phys. Rev. Lett. 79, 2348 (1997).

${ }^{39}$ M. Spannuth and J. C. Conrad, Phys. Rev. Lett. 109, 028301 (2012).

${ }^{40}$ A. Reinmüller, T. Palberg, and H. J. Schöpe, Rev. Sci. Instrum. 84, 063907 (2013).

${ }^{41}$ H. B. Eral, D. van den Ende, F. Mugele, and M. H. G. Duits, Phys. Rev. E 80, 061403 (2009).

${ }^{42}$ K. Nygård, D. K. Satapathy, J. Buitenhuis, E. Perret, O. Bunk, C. David, and J. F. van der Veen, Europhys. Lett. 86, 66001 (2009).

${ }^{43}$ T. Franosch, S. Lang, and R. Schilling, Phys. Rev. Lett. 109, 240601 (2012); 110, 059901(E) (2013) (erratum).

${ }^{44}$ J. P. Hansen and I. R. McDonald, Theory of Simple Liquids (Academic Press, New York, 2006).

${ }^{45}$ D. Henderson, Fundamentals of Inhomogeneous Fluids (Dekker, New York, 1992).
${ }^{46}$ S. Lang, R. Schilling, V. Krakoviack, and T. Franosch, Phys. Rev. E 86, 021502 (2012).

${ }^{47}$ W. R. Smith, D. Henderson, and J. A. Barker, J. Chem. Phys. 55, 4027 (1971).

${ }^{48}$ P. Schofield, Proc. Phys. Soc. 88, 149 (1966).

${ }^{49}$ J. L. Lebowitz and J. K. Percus, Phys. Rev. 122, 1675 (1961).

${ }^{50}$ D. Henderson, L. Blum, and J. L. Lebowitz, J. Electroanal. Chem. Interfacial Electrochem. 102, 315 (1979).

${ }^{51}$ R. Roth, J. Phys.: Condens. Matter 22, 063102 (2010).

${ }^{52} \mathrm{H}$. Green, Struktur der Flüssigkeiten, Handbuch der Physik Vol. 10 (Springer, Berlin/Heidelberg, 1960).

${ }^{53}$ B. A. Lowry, H. T. Davis, and S. A. Rice, Phys. Fluids 7, 402 (1964).

${ }^{54}$ O. Bunk, A. Diaz, F. Pfeiffer, C. David, C. Padeste, H. Keymeulen, P. R. Willmott, B. D. Patterson, B. Schmitt, and D. K. Satapathy, Phys. Rev. E 75, 021501 (2007). 